\title{
Autobiographical memory: a candidate latent vulnerability mechanism for psychiatric disorder following childhood maltreatment
}

\author{
Eamon J. McCrory, Vanessa B. Puetz, Eleanor A. Maguire, Andrea Mechelli, Amy Palmer, \\ Mattia I. Gerin, Philip A. Kelly, lakovina Koutoufa and Essi Viding
}

\section{Background}

Altered autobiographical memory (ABM) functioning has been implicated in the pathogenesis of depression and posttraumatic stress disorder and may represent one mechanism by which childhood maltreatment elevates psychiatric risk.
Aims
To investigate the impact of childhood maltreatment on ABM functioning.

\section{Method}
Thirty-four children with documented maltreatment and 33 matched controls recalled specific ABMs in response to emotionally valenced cue words during functional magnetic resonance imaging.

\section{Results}
Children with maltreatment experience showed reduced hippocampal and increased middle temporal and parahippocampal activation during positive ABM recall

compared with peers. During negative ABM recall they exhibited increased amygdala activation, and greater amygdala connectivity with the salience network.

\section{Conclusions}

Childhood maltreatment is associated with altered ABM functioning, specifically reduced activation in areas encoding specification of positive memories, and greater activation of the salience network for negative memories. This pattern may confer latent vulnerability to future depression and posttraumatic stress disorder.

\section{Declaration of interest}

None.

\section{Copyright and usage}

(c) The Royal College of Psychiatrists 2017. This is an open access article distributed under the terms of the Creative commons Attribution (CC BY) licence.
Childhood maltreatment is associated with a significantly increased risk of psychiatric disorder, including depression and post-traumatic stress disorder (PTSD). ${ }^{1}$ Despite a growing body of research documenting neurocognitive correlates of abuse and neglect ${ }^{2}$ there remains a relative paucity of mechanistic accounts postulating how maltreatment alters biological and neurocognitive systems in ways that can embed latent vulnerability to psychiatric disorders across the lifespan. ${ }^{3}$ Latent vulnerability refers to the way in which neurocognitive and biological systems adapt to early adverse environments in ways that may confer short-term functional advantages, but increase future risk of poor psychiatric outcome. ${ }^{3}$ The presence of latent vulnerability in itself does not mean that future psychiatric disorder is inevitable. Rather, interaction with other risk and protective factors (including genetic and social factors), as well as future stressor exposure, is thought to determine outcome. ${ }^{4}$ To date neurocognitive studies have focused on altered affect processing in maltreated individuals, including studies of threat reactivity and stress/emotion regulation, as well as reward processing. ${ }^{4-7}$ However, no neurocognitive studies to date have investigated the very human phenomenon of personal memory.

Autobiographical memory (ABM) refers to the recollection of personally experienced events. It is thought to play a central role in scaffolding our sense of self and grounding our ability to remain oriented in the present. ${ }^{8}$ Individuals with a range of psychiatric disorders, in particular those with PTSD and depression, present with an overgeneral memory (OGM) pattern, characterised by thematic or categorical description, with a relative dearth of detail. ${ }^{9}$ Not only is OGM observed in individuals with existing clinically diagnosed affective disorders, it predicts symptoms of PTSD $^{10}$ and depression in at-risk individuals. For example, in a 1-year longitudinal study OGM was found to predict the onset of depressive disorder in adolescents at familial risk for depression. ${ }^{11}$ Such findings are consistent with the notion that OGM is implicated in the pathogenesis of psychiatric disorder. ${ }^{9,10}$

OGM has been documented in those who have experienced trauma (including maltreatment) during childhood. ${ }^{12,13}$ It has been proposed that such OGM processing emerges, at least in part, because traumatic material is aversive and prompts an avoidant recall style. ${ }^{9}$ No study to date has investigated the functional neural correlates of ABM in children who have been maltreated; as such we do not know whether these children engage a typical network of brain areas during ABM processing, or how differential engagement of this network during positive and negative memory recall may contribute to psychiatric risk.

The primary aim of the current study was therefore to examine both neurocognitive and behavioural indices of $A B M$ in a community sample of children with documented experiences of abuse and neglect. Based on previous research, ${ }^{13}$ we predicted that the maltreated group would show OGM at a behavioural level compared with their non-maltreated peers, and that such a pattern would still be observed even if any children presenting with a current psychiatric disorder were excluded. At a neural level, our predictions were informed by adult studies, in the absence of any prior imaging studies of $A B M$ in children. In typical adults, ABM processing engages a network of prefrontal, parietal and temporal regions including the ventromedial prefrontal cortex (vmPFC), middle temporal gyrus (MTG), hippocampus and temporoparietal junction (TPJ), ${ }^{14}$ and the richness of ABM recall is associated with hippocampal activation. ${ }^{15}$ Clinical studies of adult patients with PTSD and depression have reported that although these individuals engage core components of the ABM 
network, they differentially weight regions within this network during specific $A B M$ recall, depending on the task. ${ }^{16,17}$ In light of these adult findings, we predicted that the core ABM network would be engaged by both groups of children, with altered activity of the medial temporal lobe during ABM recall in those who had experienced maltreatment. Depression, a common outcome associated with maltreatment, has been associated with impaired processing of positive information. ${ }^{17}$ As such, we predicted reduced hippocampal activation during positive relative to negative ABM recall, given that richness of $\mathrm{ABM}$ recall is associated with hippocampal activation. ${ }^{13}$ Furthermore, in view of the extant evidence indicating heightened amygdala response to negative stimuli in children who have been maltreated ${ }^{4-5}$ and in adults at greater risk of depression during negative $A B M$ recall, ${ }^{18}$ we also predicted greater amygdala activation during negative $\mathrm{ABM}$ recall alongside greater functional connectivity between the amygdala and regions implicated in salience processing.

\section{Method}

\section{Participants}

A total of 67 children aged 10-14 years were recruited for the study: 34 with a documented experience of maltreatment (maltreatment group) recruited via a London social services department $(n=27)$ and post-adoption services $(n=7)$ and 33 non-maltreated children with no prior social services contact (non-maltreatment group) recruited via schools and community advertisements. Participants were matched across a range of domains, including gender, socioeconomic status, IQ and pubertal status (Table 1) to increase the likelihood that any observed group differences in ABM functioning could be attributable to maltreatment experience. Standard exclusion criteria for all participants included pervasive developmental disorder, neurological abnormalities, standard magnetic resonance imaging (MRI) contraindications and IQ $<70$. Groups did not differ significantly in age, pubertal status, gender, handedness, IQ, reading ability, socioeconomic status and ethnicity (Table 1). An additional 19 children were originally recruited but subsequently excluded from analysis because of one or more of the following reasons: excess motion (more than $10 \%$ of data $>1.5 \mathrm{~mm}$ in both runs, $n=3$ ), less than $70 \%$ of memories remembered in the scanner $(n=14)$, technical issues $(n=4)$. There were no differences between the groups in the number of participants per exclusion category (all $P$-values $>0.22$ ). Consent was obtained from the child's legal guardian and assent was obtained from all children. All procedures were approved by UCL Research Ethics Committee (0895/002).

\section{Measures}

\section{Maltreatment history}

All the participants in the maltreatment group had experienced a level of maltreatment requiring social services referral. Severity of neglect, emotional, sexual and physical abuse as well as intimate partner violence, were rated by the child's social worker or adoptive parent on the basis of social services file information. Ratings from zero (not present) to four (severe) were applied to each category. ${ }^{19}$ The majority of children had been exposed to neglect, emotional abuse and intimate partner violence (online Table DS1). In addition, children completed the Childhood Trauma Questionnaire (CTQ, Table 1 and see online Table DS2 for the scores on the subscales of the CTQ). ${ }^{20}$

\section{Psychiatric symptomatology}

The Strengths and Difficulties Questionnaire (SDQ, parent rated $)^{21}$ was used to characterise overall behavioural and psychological functioning that could be related in our exploratory

Table 1 Background data, including cognitive and Autobiographical Memory Test performance and psychiatric status in the maltreated and non-maltreated groups

\begin{tabular}{|c|c|c|c|}
\hline & $\begin{array}{l}\text { Maltreatment } \\
\text { group }(n=34)\end{array}$ & $\begin{array}{l}\text { Non-maltreatment } \\
\text { group }(n=33)\end{array}$ & $P$ \\
\hline Gender, female: $n(\%)$ & $17(50)$ & $19(58)$ & 0.620 \\
\hline Ethnicity, White: $n(\%)$ & $23(68)$ & $17(52)$ & 0.220 \\
\hline Socioeconomic status, parental education, $n(\%)$ beyond secondary & $14(41)$ & $13(39)$ & 0.802 \\
\hline Psychiatric diagnoses, ${ }^{\text {a }} n(\%)$ & 7 (23) & $0(0)$ & 0.004 \\
\hline Age, years: mean (s.d.) & $12.53(1.60)$ & $12.66(1.29)$ & 0.730 \\
\hline Pubertal status, ${ }^{b}$ mean (s.d.) & $2.83(1.13)$ & $2.63(0.63)$ & 0.401 \\
\hline Wechsler Abbreviated Scales of Intelligence, IQ: mean (s.d.) & $105.03(15.08)$ & $108.75(12.01)$ & 0.270 \\
\hline Reading score mean (s.d.) & $117.88(20.42)$ & $117.55(17.66)$ & 0.956 \\
\hline Visual memory, number of errors: ${ }^{c}$ mean (s.d.) & $12.45(6.96)$ & $9.49(6.54)$ & 0.080 \\
\hline Verbal memory, number correct: ${ }^{c}$ mean (s.d.) & $25.65(4.92)$ & $26.58(3.64)$ & 0.390 \\
\hline Childhood Trauma Questionnaire, total score: mean (s.d.) & $38.15(14.75)$ & $28.91(4.77)$ & 0.001 \\
\hline Strength and Difficulties Questionnaire - parent report, total difficulties score: mean (s.d.) & $13.34(7.38)$ & $7.06(4.70)$ & $<0.001$ \\
\hline Emotional symptoms score & $3.23(2.66)$ & $1.71(1.60)$ & 0.007 \\
\hline Conduct problems score & $2.94(2.18)$ & $0.71(1.01)$ & 0.024 \\
\hline Hyperactivity score & $5.16(2.68)$ & $2.16(1.59)$ & 0.002 \\
\hline Peer problems score & $2.35(2.01)$ & $1.03(1.17)$ & 0.003 \\
\hline Prosocial behaviour score & $7.52(2.14)$ & $8.94(1.53)$ & 0.010 \\
\hline Autobiographical Memory Test, total: mean (s.d.) & $3.48(2.45)$ & $2.00(2.02)$ & 0.017 \\
\hline Positive memories & $1.58(1.34)$ & $0.96(1.11)$ & 0.066 \\
\hline Negative memories & $1.90(1.49)$ & $1.04(1.11)$ & 0.018 \\
\hline \multicolumn{4}{|c|}{$\begin{array}{l}\text { a. Child mental health symptoms were assessed with the Development and Well-Being Assessment (DAWBA). Information on the DAWBA was missing for one child in the } \\
\text { non-maltreatment group and for four children in the maltreatment group. Seven children in the maltreatment group met diagnostic criteria: oppositional defiant disorder (ODD) } n=4 \\
\text { conduct disorder } n=1 \text {; attention-deficit hyperactivity disorder } n=1 \text {; generalised anxiety disorder } n=1 \text {; separation anxiety disorder (SAD) } n=1 \text {. Note one child was comorbid for ODD } \\
\text { and SAD. } \\
\text { b. Composite score of self-report and parent rating of Puberty Development Scale (PDS). } \\
\text { c. Measured with the CogState cognitive assessment system. }\end{array}$} \\
\hline
\end{tabular}


analyses to observed patterns of neural functioning. The Development and Well-Being Assessment (DAWBA) ${ }^{22}$ was used to assess psychiatric status by an experienced clinician (E.M.C.) masked to group status; this permitted neuroimaging analyses that included and excluded those with presenting psychiatric disorder, reducing the likelihood that any observed differences could be attributable to psychiatric status (Table 1).

\section{Cognitive ability}

Cognitive functioning was assessed using two subscales of the Wechsler Abbreviated Scales of Intelligence, ${ }^{23}$ the word reading subscale of the Wide Range Achievement Test (WRAT 4) ${ }^{24}$ and two subtests of the CogState battery ${ }^{25}$ pertaining to visual and verbal episodic memory (Table 1).

\section{Autobiographical Memory Test (AMT) and function MRI (fMRI) stimulus generation}

The AMT, ${ }^{26}$ a standard measure of OGM, was administered to all participants in a session 1-3 weeks before scanning. Participants generated specific memories in response to ten positive (for example 'achieve', 'caring') and ten negative cue words (for example 'mistake', 'lonely'). OGMs were defined as 'memories that did not contain at least one specific detail that identifies an event as a distinct episode. ${ }^{13,27}$ See online supplement DS1 for a description of the kinds of memories recalled.

Stimuli for the fMRI task included specific memories generated from the AMT and specific memories generated from supplemental valenced cue words, administered to ensure a full set of 20 specific memories were generated (10 positive ABMs, 10 negative $\mathrm{ABMs}$ ) for the imaging component, in line with prior fMRI studies. ${ }^{28,29}$ These memories were rephrased by the participants in 3-4 words to prompt recall during the scanning session. Also in line with prior studies in adults, ${ }^{28}$ a control task was employed in which participants were presented with pictures and brief descriptions of ten everyday objects. Participants were given $20 \mathrm{~s}$ to memorise each object. As with the ABMs, key features were captured in brief sentence cues for presentation during fMRI.

\section{MRI scanning session}

There were three memory conditions: positive ABM recall, negative $A B M$ recall and object recall. The fMRI task consisted of two runs of 15 trials, each comprising 5 positive ABMs, 5 negative ABMs and 5 object memories presented in a pseudorandomised order, which prevented the consecutive presentation of a stimulus category. Each trial began with a sentence cue. Participants indicated successful or unsuccessful memory retrieval via a self-paced button press. The text cue then disappeared and the word 'Elaborate' was presented during which time the participants recalled the memory or object (cue+retrieval+ elaborate $=20 \mathrm{~s}$ ). The elaboration period was followed by an audio tone $(1 \mathrm{~s})$, then a five-point Likert scale rating recall difficulty $(4.5 \mathrm{~s})$ and a second rating of vividness of the recalled memory $(4.5 \mathrm{~s})$. The participants could provide the ratings at any time during the $4.5 \mathrm{~s}$ rating periods. Any excess time related to the period after the button press for the second rating was added to the subsequent inter-trial interval period of $6.5 \mathrm{~s}$ (thus introducing jitter). Post-scanning, ABMs were rated on emotional salience, remoteness and agency (online Table DS3).

\section{fMRI data acquisition}

All data were acquired on a 1.5 tesla Siemens (Siemens Medical Systems, Erlangen, Germany) Avanto MRI scanner with a 32channel head coil during two runs of approximately 9 min each.
During each run, a total of $181 T_{2}{ }^{*}$-weighted echo-planar (EPI) volumes were acquired, covering the whole brain with the following acquisition parameters: slice thickness: $2 \mathrm{~mm}$; repetition time $(\mathrm{TR})=85 \mathrm{~ms} ; \quad$ echo time $\quad(\mathrm{TE})=50 \mathrm{~ms}$; field of view $($ FOV $)=192 \mathrm{~mm} \times 192 \mathrm{~mm}^{2} ; 35$ slices per volume, gap between slices: $1 \mathrm{~mm}$; flip angle: $90^{\circ}$ ). A high-resolution, three-dimensional $T_{1}$-weighted structural scan was acquired with a magnetisationprepared rapid gradient echo sequence. Imaging parameters were: 176 slices; slice thickness: $1 \mathrm{~mm}$; gap between slices: $0.5 \mathrm{~mm}$; $\mathrm{TE}=2730 \mathrm{~ms} ; \mathrm{TR}=3.57 \mathrm{~ms} ; \mathrm{FOV}=256 \mathrm{~mm} \times 256 \mathrm{~mm}^{2} ;$ matrix size: $256 \times 256$; voxel size: $1 \times 1 \times 1 \mathrm{~mm}$ resolution.

\section{Analyses}

\section{Behavioural analyses}

For the AMT, a repeated-measures ANOVA was performed with group as the between-participant factor and number of OGMs recalled as the within-participant factor to investigate group differences in OGM recall. For ABM recall in the scanner, repeated measure ANOVAs were performed in relation to: (a) recall success; and (b) the ratings of difficulty and vividness (see online supplement DS3 for details).

\section{fMRI analyses}

Data analyses were conducted using the software package SPM8 (www.fil.ion.ucl.ac.uk/spm/software/spm8) implemented in Matlab 2015a. After discarding the first three volumes of each run to allow for $T_{1}$ equilibration effects, data were realigned; initially within each run and then across the two runs to the first image of the first run. Data were normalised into Montreal Neurological Institute (MNI) space using deformation fields from $T_{1}$ scan segmentation at a voxel size of $3 \times 3 \times 3 \mathrm{~mm}$. The resulting images were smoothed with a $6 \mathrm{~mm}$ Gaussian filter and high-pass filtered at $128 \mathrm{~Hz}$. Fixed-effects statistics for each individual were calculated by convolving boxcar functions modelling the three conditions with a canonical hemodynamic response function, namely: positive $\mathrm{ABM}$ retrieval/elaboration; negative $\mathrm{ABM}$ retrieval/ elaboration; object retrieval/elaboration, with a separate regressor included for the ratings.

Additionally, the six motion parameters were added to the model as regressors of no interest. In order to further minimise movement-related artefacts, images corrupted because of head motion greater than $1.5 \mathrm{~mm}$ were removed and replaced by interpolations of adjacent images ( $<7 \%$ of each participant's data). For these participants, we included an additional regressor of no interest to model the interpolated scans. Individual participants' SPMs containing the parameter estimates of the three conditions and the ratings were then entered as fixed-effects factors into a repeated-measures mixed-effects ANOVA containing a 'subject' factor for random effects for group analyses.

We first analysed main effects of $A B M$ recall across groups to explore neural networks underlying $\mathrm{ABM}$ recall in children (contrast $\mathrm{ABM}$ recall $v$. object recall). Second, to investigate the maltreatment and non-maltreatment group differences in $\mathrm{ABM}$ recall, contrast images were calculated for $\mathrm{ABM}$ recall (positive and negative) $v$. object recall. Third, to investigate whether the ABM network was differentially weighted as a function of valence, positive and negative $A B M$ recall was contrasted across groups (contrast positive $v$. negative ABM recall). Fourth, to investigate maltreatment and non-maltreatment group differences in the weighting of positive $v$. negative $\mathrm{ABM}$ recall, contrast images were calculated for positive $v$. negative $\mathrm{ABM}$ recall.

Whole brain and region of interest (ROI) analyses. Whole brain analyses were conducted using Monte-Carlo Simulation (3D 
ClusterSim $)^{30}$ correcting for multiple comparisons. Cluster-sizecorrected results are reported (voxel-wise $P<0.005$, cluster extent $(k e)=75)$ corresponding to $P=0.05$, family-wise error (FWE)corrected. All tests are two-sided. ROI analyses of the amygdala were carried out using bilateral anatomical masks that were anatomically defined using the automated anatomical labelling (AAL) atlas as implemented in the WFU PickAtlas. Correction for multiple comparisons corresponding to $P=0.05$, FWE was applied to the two amygdala ROIs for the negative $v$. positive ABM recall. The right amygdala showed the most robust difference between groups and was therefore used in all further analyses, i.e. the psychophysiological interaction (PPI) and further correlational analyses between brain regions and indices of maltreatment.

PPI analyses. A PPI analysis ${ }^{31}$ was used to assess amygdala functional connectivity. For the PPI analyses, the time-course of the right amygdala was extracted for each participant and subsequently multiplied by a vector contrasting negative $A B M$-positive ABM. Each participant's contrast images were then entered into an ANOVA in order to estimate effects at the second level. The PPI analysis was corrected in line with the whole brain analyses (voxel-wise $P<0.005, k e=75$ ).

Finally, contrast estimates were extracted from the peak voxels of clusters showing significant group differences in the contrast positive $A B M$ recall $v$. negative $A B M$ recall, i.e. the right $M T G$, right hippocampus and right amygdala using the MarsBaR Toolbox Release 0.44 (http://marsbar.sourceforge.net/) implemented in SPM. The contrast estimates were subsequently correlated with (a) indices of maltreatment (onset, severity and duration of maltreatment), and (b) total score on the SDQ in SPSS version 21

Exploratory post hoc correlational analyses within the maltreatment group were conducted to assess the relationship between differential brain activation and maltreatment experience (severity, onset and duration) as well as overall functioning as measured by SDQ total score. As shown in Table 1, children in the maltreatment group (perhaps unsurprisingly) showed greater levels of symptoms on the SDQ than their peers, indicating that although most did not reach clinical threshold, psychological and behavioural difficulties were nonetheless elevated.

\section{Results}

\section{AMT performance}

The maltreatment group generated more OGMs across conditions $(t(55)=-2.46, P=0.017,95 \% \mathrm{CI}-0.22$ to -0.02 ; see Table 1$)$. There were no significant main effects of valence or group $\times$ valence interactions. Excluding participants meeting criteria for a psychiatric disorder did not alter the pattern of findings.

\section{fMRI results}

Across groups $\mathrm{ABM} v$. object recall engaged a network of cortical midline structures including the vmPFC, posterior cingulate cortex (pCC), bilateral MTG extending into hippocampus, precuneus and TPJ (online Tables DS4 and DS5). No significant group $\times$ condition interactions were found for either $\mathrm{ABM}$ recall $>$ object recall or object recall $>A B M$ recall. Analyses of regions more active during positive $v$. negative $\mathrm{ABM}$ recall across the whole sample revealed engagement of the left hippocampus, whereas the opposite contrast yielded no significant differences (Table 2).

Between group comparisons at the whole brain level revealed a group $\times$ valence interaction for emotion (Table 2). This interaction was driven by greater activation for positive than negative $\mathrm{ABM}$ in the non-maltreatment group in the right hippocampus extending into the right fusiform gyrus and inferior temporal gyrus and the reverse trend in the maltreatment group (Fig. 1). Conversely, the maltreatment group, relative to the non-maltreatment group, showed increased activity in the right MTG extending into the parahippocampal gyrus during positive as compared with negative ABM recall (Fig. 1). Again, excluding participants meeting criteria for psychiatric disorder did not alter this pattern of findings.

In line with our predictions, the ROI-analysis indicated greater bilateral amygdala activation for negative than positive $\mathrm{ABM}$ in

\begin{tabular}{|c|c|c|c|c|c|c|c|}
\hline Participant contrast and brain region & Right/left & $x$ & $y$ & $z$ & ke & $t$ & $Z$ \\
\hline \multicolumn{8}{|c|}{$\begin{array}{l}\text { Whole brain contrast for positive } A B M \text { recall > negative } A B M \text { recall } \\
\text { All participants }\end{array}$} \\
\hline \multirow[t]{3}{*}{ Hippocampus } & Left & -21 & -25 & -20 & \multirow[t]{3}{*}{80} & 4.67 & 4.58 \\
\hline & Left & -27 & -37 & -17 & & 3.32 & 3.29 \\
\hline & Left & -33 & -16 & -20 & & 3.15 & 3.12 \\
\hline \multicolumn{8}{|l|}{ Non-maltreatment group $>$ maltreatment group } \\
\hline Hippocampus & Right & 33 & -19 & -11 & \multirow[t]{3}{*}{384} & 5.16 & 5.03 \\
\hline \multirow[t]{2}{*}{ Inferior temporal gyrus extending into fusiform gyrus } & Right & 39 & -43 & -8 & & 4.50 & 4.42 \\
\hline & Right & 36 & -58 & -5 & & 4.50 & 4.41 \\
\hline \multicolumn{8}{|l|}{ Maltreatment group $>$ non-maltreatment group } \\
\hline \multirow[t]{2}{*}{ Middle temporal gyrus } & Right & 51 & -16 & -20 & \multirow[t]{3}{*}{186} & 4.48 & 4.39 \\
\hline & Right & 45 & -4 & -26 & & 4.3 & 4.22 \\
\hline Parahippocampal gyrus & Right & 33 & -19 & -23 & & 3.99 & 3.93 \\
\hline \multicolumn{8}{|c|}{$\begin{array}{l}\text { Region of interest contrast for negative } A B M \text { recall > positive } A B M \text { recall } \\
\text { Maltreatment group }>\text { non-maltreatment group }\end{array}$} \\
\hline Amygdala $a^{a}$ & Right & 27 & -4 & -14 & 5 & 3.23 & 3.20 \\
\hline \multicolumn{8}{|l|}{$\begin{array}{l}\text { PPI contrast for the negative } A B M \text { recall }>\text { positive } A B M \text { recall } \\
\text { Maltreatment group }>\text { non-maltreatment group }\end{array}$} \\
\hline \multirow[t]{3}{*}{ Middle anterior cingulate cortex } & Right & 9 & 38 & 43 & \multirow[t]{3}{*}{112} & 3.82 & 3.59 \\
\hline & Right & 15 & 26 & 31 & & 3.58 & 3.38 \\
\hline & Right & 9 & 32 & 34 & & 3.56 & 3.37 \\
\hline
\end{tabular}




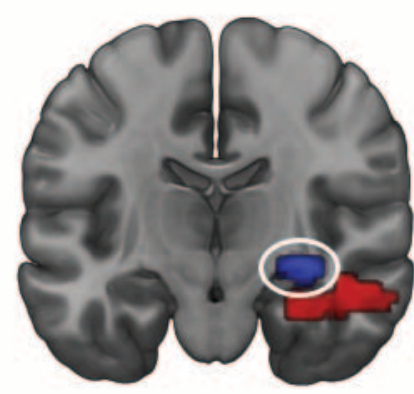

(b)

(c)

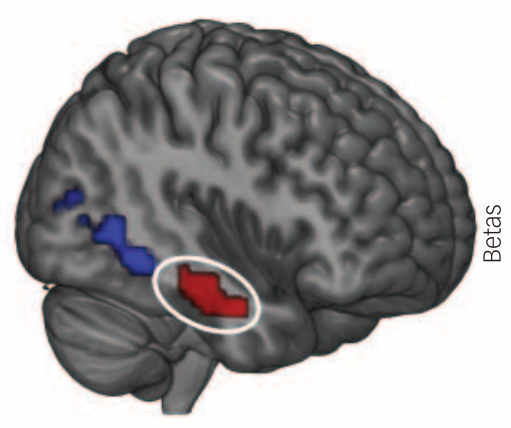

Right hippocampus
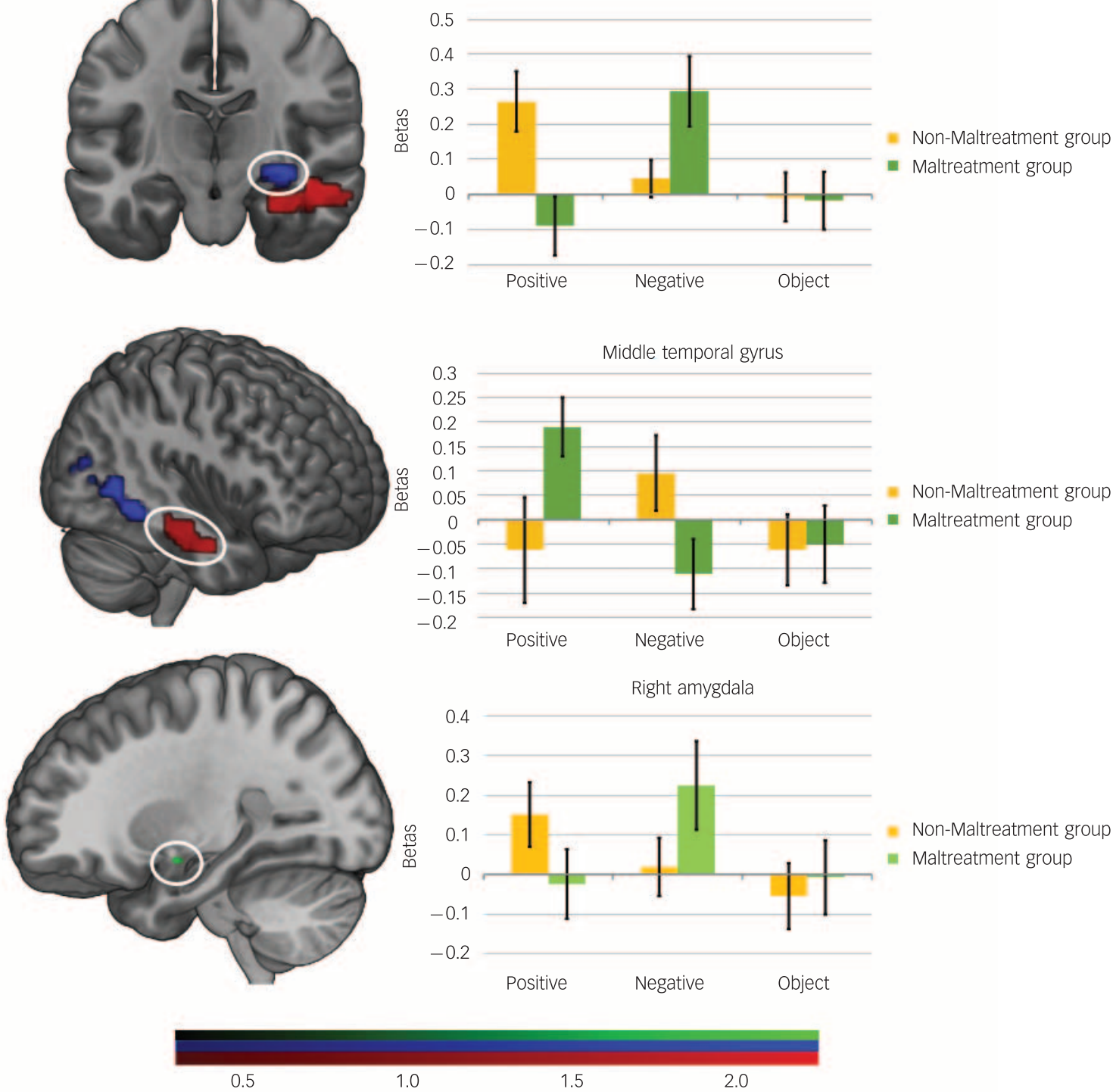

0.5

1.5

Fig. 1 Group differences in haemodynamic activity during autobiographical memory (ABM) recall in response to positive $v$. negative cues.

(a) Whole-brain group analyses showing significantly reduced activation in the maltreated group relative to the non-maltreated group (controls) in response to the positive ABM recall $v$. negative ABM recall in the right hippocampus (blue) and (b) higher activation in the middle temporal gyrus (MTG; red). (c) Regions of interest analyses showing greater right

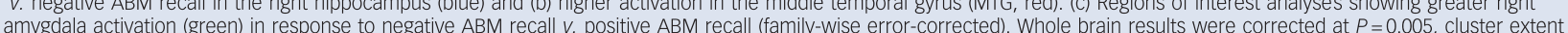
$(\mathrm{Ke})=75$ and parameter estimates represent differences in activation between positive and negative ABM recall. Error bars indicate one standard error of the mean (SEM).

the maltreatment group relative to the non-maltreatment group (right $P=0.013$, left $P=0.042$, FWE-corrected) (Fig. 1 and Table 2). The PPI analysis indicated that during negative $v$. positive $A B M$ recall the maltreatment group relative to the nonmaltreatment group displayed greater functional connectivity between the right amygdala and right middle anterior cingulate cortex (mACC).

Exploratory post hoc correlations in those regions showing differential group activation during $\mathrm{ABM}$ recall indicated that MTG activity during positive ABM recall correlated negatively with severity of maltreatment (Kaufman) $\left(r_{s}=-0.35, P=0.04\right)$ and average duration of maltreatment $(r=-0.54, P=0.001$; see online Fig. DS1). No significant associations were found in relation to maltreatment onset. Overall behavioural/psychological functioning as measured by the SDQ total score correlated positively with hippocampal $(r=0.47, P=0.007)$ and amygdala $(r=0.36, P=0.049)$ activation during negative memory recall in the maltreatment group.
No group differences or interactions were found in relation to recall success, or in-scanner/post-scanner ratings with the exception of a group $\times$ valence interaction for emotion. Those in the non-maltreatment group reported that they felt significantly more negative than the maltreatment group during negative $\mathrm{ABM}$ recall $(t(58)=-2.72, P=0.009$; online supplement DS3).

\section{Discussion}

\section{Main findings}

To our knowledge, the present study is the first to demonstrate that maltreatment experience is associated with altered neural responses during $\mathrm{ABM}$ recall. Specifically, when positive and negative conditions were contrasted, children who had been maltreated compared with their non-maltreated peers, showed reduced activation in the right hippocampus, extending into the inferior temporal and fusiform gyri but increased activation in 
the right middle temporal and parahippocampal gyri. As predicted, increased amygdala activation and functional connectivity with the anterior cingulate cortex-related salience network was observed when children who had been maltreated recalled negative compared with positive memories. At the behavioural level, maltreatment was associated with greater OGM recall for both positive and negative cue words, even when children meeting diagnostic threshold were excluded. This suggests that the previously observed pattern of OGM in maltreated samples was not the result of undiagnosed psychopathology.

Relative to a standard control condition of object recall, specific ABM recall across child participants during fMRI engaged the same network previously observed in healthy adult samples, including the vmPFC, pCC, MTG, hippocampus, temporal pole and TPJ. This suggests comparability in the neural basis of ABM processing across children and adults. Contrasting the recall of positive and negative $\mathrm{ABMs}$, revealed that the maltreated group showed altered neural activation during recall of positive and negative memories relative to their peers.

First, we observed reduced activation of the right hippocampus during positive relative to negative memory recall. Given the role of the hippocampus in processing sensory and perceptual details as well as encoding temporal context, this attenuation in neural response in children who had been maltreated may reflect more poorly specified reconstruction of positive memories in these individuals. ${ }^{32}$ By contrast, we observed increased activation of the amygdala during negative $v$. positive memory recall in the maltreated group in addition to increased functional connectivity of the amygdala with regions implicated in salience processing. This suggests that children who have been maltreated accord heightened salience to negative memories relative to their peers. Our exploratory post hoc correlations were consistent with these findings. Both hippocampal and amygdala activation during negative recall in the maltreatment group was greatest in those individuals with the highest levels of psychological and behavioural problems as indexed by the SDQ.

\section{Comparison with findings from other studies}

These findings are also in line with several adult studies of depression ${ }^{17}$ and PTSD. ${ }^{16}$ For example, St Jacques and colleagues report elevated amygdala and hippocampal response in patients with PTSD during construction of emotionally intense ABMs. ${ }^{16}$ Similarly, in a study of patients with current depression, remitted depression and individuals at high familial risk of depression, Young and colleagues (2016) reported increased amygdala activation, and functional connectivity with regions implicated in salience processing (including the dorsal anterior cingulate cortex) during negative $\mathrm{ABM}$ recall in all three groups relative to healthy controls. ${ }^{18}$ It was concluded that heightened amygdala activation and functional connectivity with the salience network during negative $\mathrm{ABM}$ recall may therefore represent a trait-like marker of depression. ${ }^{18}$ Our findings in children who had been maltreated, whom we know to be at an elevated risk of developing depression in adulthood, are consistent with this view.

\section{Interpretation of our findings}

We suggest that the current findings may reflect increased latent vulnerability to psychiatric disorder in two main ways. First, greater OGM may reduce the ability of children who have been maltreated to draw on past experiences in order to effectively negotiate future stressors. $\mathrm{ABM}$ recall is believed to be necessary to generate adaptive responses to social interactions and stressful circumstances, playing a role in simulating future events. ${ }^{33}$
Furthermore, OGM has been associated with reduced problemsolving in depression ${ }^{9}$ and PTSD. ${ }^{34}$ Second, decreased specificity and salience of positive, relative to negative, memories in children who have experienced maltreatment may increase the likelihood of a negative inferential style, negative self-schemata and a ruminative response style, all cognitive vulnerabilities associated with maltreatment ${ }^{35-38}$ and central to cognitive theories of depression and other psychiatric disorders.

\section{Limitations}

A number of limitations of this study should be noted. First, because of the cross-sectional design, it was not possible to examine the developmental trajectories of altered ABM processing. Future studies employing longitudinal designs should examine whether altered behavioural and/or neural processing of negative and positive ABMs predicts future psychopathology (or maladaptive functioning) in individuals with histories of maltreatment in childhood. Second, because of our sample size, we were unable to examine the influence of gender, although there is suggestion from previous research that there may be some differential mental health outcomes for boys and girls exposed to early adversity. We also did not have sufficient power to investigate whether experiences of neglect or experiences of abuse were driving the observed differences; in our sample these forms of maltreatment frequently co-occurred (online Table DS1). Third, although we employed an established task to assess ABM functioning in the scanner, other paradigms have employed a verbal rather than a visual control condition, which may provide a more stringent baseline. ${ }^{17}$

\section{Implications}

The present results demonstrate that children who have experienced maltreatment are characterised by a pattern of OGM and altered neural processing of positive and negative memories. This pattern of processing may instantiate latent vulnerability to psychiatric disorder. Longitudinal studies are required to test this hypothesis further, and could also examine whether altered processing presents in the same way, even in the presence of a disorder. The current findings also raise the prospect that $\mathrm{ABM}$ functioning may represent a potential target for preventative intervention for children who have experienced maltreatment prior to the onset of psychiatric disorders for which these children are at increased risk. ${ }^{3-9}$.

\footnotetext{
Eamon J. Mccrory, PhD, DClin Psy, Vanessa B. Puetz, PhD, Division of Psychology and Language Sciences, University College London, London, and The Anna Freud Centre, London, UK; Eleanor A. Maguire, PhD, Wellcome Trust Centre for Neuroimaging, Institute of Neurology, University College London, London, UK; Andrea Mechelli, PhD, Department of Psychosis Studies, Institute of Psychiatry, Psychology \& Neuroscience, King's College London, London, UK; Amy Palmer, PhD, Mattia I. Gerin, MSc, Philip A. Kelly, PhD, Iakovina Koutoufa, MSC, Division of Psychology and Language Sciences, University College London, London, and The Anna Freud Centre, London, UK; Essi Viding, PhD, Division of Psychology and Language Sciences, University College London, London, UK

Correspondence: Eamon J. McCrory, Division of Psychology and Language Sciences, University College London, Gower Street, London WC1 6BT, UK. Email: e.mccrory@ucl.ac.uk

First received 23 Dec 2016, final revision 25 May 2017, accepted 1 Jun 2017
}

\section{Funding}

This work was funded by a grant to E.J.M. from the UK Economic and Social Research Council (ES/KO05723/1). E.A.M. is supported by a Wellcome Trust Principal Research Fellowship (101759/Z/13/Z). 


\section{References}

1 Gilbert R, Widom CS, Browne K, Fergusson D, Webb E, Janson S. Burden and consequences of child maltreatment in high-income countries. Lancet 2009; 373: $68-81$.

2 McCrory EJ, Gerin MI, Viding E. Annual Research Review: Childhood maltreatment, latent vulnerability and the shift to preventative psychiatry - the contribution of functional brain damage. J Child Psychol Psychiatry 2017; 58: 338-57.

3 McCrory EJ, Viding E. The theory of latent vulnerability: reconceptualizing the link between childhood maltreatment and psychiatric disorder. Dev Psychopathol 2015; 27: 493-505.

4 Mccrory EJ, De Brito SA, Sebastian CL, Mechelli A, Bird G, Kelly PA, et al. Heightened neural reactivity to threat in child victims of family violence. Curr Biol 2011; 21: 947-8.

5 Mccrory EJ, De Brito SA, Kelly PA, Bird G, Sebastian CL, Mechelli A, et al. Amygdala activation in maltreated children during pre-attentive emotional processing. Br J Psychiatry 2013; 202: 269-76.

6 Hanson JL, Hariri AR, Williamson DE. Blunted ventral striatum development in adolescence reflects emotional neglect and predicts depressive symptoms Biol Psychiatry 2015; 78: 598-605.

7 McLaughlin KA, Peverill M, Gold AL, Alves S, Sheridan MA. Child maltreatment and neural systems underlying emotion regulation. J Am Acad Child Adolesc Psychiatry 2015; 54: 753-62.

8 Conway MA, Pleydell-Pearce CW. The construction of autobiographical memories in the self-memory system. Psychol Rev 2000; 107: 261-88.

9 Dalgleish T, Werner-Seidler A. Disruptions in autobiographical memory processing in depression and the emergence of memory therapeutics. Trends Cogn Sci 2014; 18: 596-604.

10 Kleim B, Ehlers A. Reduced autobiographical memory specificity predicts depression and posttraumatic stress disorder after recent trauma. Int $J$ Emerg Ment Health 2008; 10: 158-60.

11 Rawal A, Rice F. Examining overgeneral autobiographical memory as a risk factor for adolescent depression. J Am Acad Child Adolesc Psychiatry 2012 51: $518-27$

12 Hitchcock C, Nixon RD V, Weber N. A review of overgeneral memory in child psychopathology. Br J Clin Psychol 2014; 53: 170-93.

13 Valentino K, Toth SL, Cicchetti D. Autobiographical memory functioning among abused, neglected, and nonmaltreated children: the overgeneral memory effect. J Child Psychol Psychiatry 2009; 50: 1029-38.

14 Martinelli P, Sperduti M, Piolino P. Neural substrates of the self-memory system: new insights from a meta-analysis. Hum Brain Mapp 2013; 34 1515-29.

15 Gilboa A, Shalev AY, Laor L, Lester $\mathrm{H}$, Louzon $\mathrm{Y}$, Chisin $\mathrm{R}$, et al. Functional connectivity of the prefrontal cortex and the amygdala in posttraumatic stress disorder. Biol Psychiatry 2004; 55: 263-72.

16 St Jacques PL, Kragel PA, Rubin DC. Neural networks supporting autobiographical memory retrieval in posttraumatic stress disorder. Cogn Affect Behav Neurosci 2013; 13: 554-66.

17 Young KD, Bellgowan PSF, Bodurka J, Drevets WC. Behavioral and neurophysiological correlates of autobiographical memory deficits in patients with depression and individuals at high risk for depression. JAMA Psychiatry 2013; 70: 698-708.
18 Young KD, Drevets WC, Bodurka J, Preskorn SS. Amygdala activity during autobiographical memory recall as a biomarker for residual symptoms in patients remitted from depression. Psychiatry Res 2016; 248: 159-61.

19 Kaufman J, Jones B, Stieglitz E, Vitulano L, Mannarino AP. The use of multiple informants to assess children's maltreatment experiences. I Fam violence 1994; 9 : 227-48.

20 Bernstein DP, Stein JA, Newcomb MD, Walker E, Pogge D, Ahluvalia T, et al. Development and validation of a brief screening version of the Childhood Trauma Questionnaire. Child Abus Negl 2003; 27: 169-90.

21 Goodman R. The Strengths and Difficulties Questionnaire: a research note. J Child Psychol Psychiatry 1997; 38: 581-6.

22 Goodman R, Ford T. The Development and Well-Being Assessment: description and initial validation of an integrated assessment of child and adolescent psychopathology. J Child Psychol Psychiatry 2000; 41: 645-55.

23 Wechsler D. Wechsler Memory Scale (WMS-III). Psychological Corporation, 1997.

24 Jastak JF, Wilkinson G. Wide Range Achievement Test: Manual of Instructions (4th edn). Jastak Associates, 1984.

25 Maruff $P$, Lim YY, Darby D, Ellis KA, Pietrzak RH, Snyder PJ, et al. Clinical utility of the cogstate brief battery in identifying cognitive impairment and Alzheimer's disease. BMC Pharmacol Toxicol 2013; 1: 30

26 Williams JM, Broadbent K. Autobiographical memory in suicide attempters. J Abnorm Psychol 1986; 95: 144-9.

27 Johnson RJ, Greenhoot AF, Glisky E, McCloskey LA. The relations among abuse, depression, and adolescents' autobiographical memory. J Clin Child Adolesc Psychol 2005; 34: 235-47.

28 Summerfield JJ, Hassabis D, Maguire EA. Cortical midline involvement in autobiographical memory. Neuroimage 2009; 44: 1188-200.

29 Hassabis D, Kumaran D, Maguire EA. Using imagination to understand the neural basis of episodic memory. J Neurosci 2007; 27: 14365-74.

30 Ward B. Simultaneous Inference for FMRI Data. AFNI 3dDeconvolve Documentation. Medical College of Wisconsin, 2000.

31 Friston K, Buechel C, Fink G, Morris J, Rolls E, Dolan R. Psychophysiological and modulatory interactions in neuroimaging. Neuroimage 1997; 6: 218-29.

32 Maguire EA, Mullally SL. The hippocampus: a manifesto for change. J Exp Psychol Gen 2013; 142: 1180-9.

33 Williams JMG, Ellis NC, Tyers C, Healy H, Rose G, Macleod AK. The specificity of autobiographical memory and imageability of the future. Mem Cognit 1996; 24: 116-25.

34 Sutherland K, Bryant RA. Social problem solving and autobiographical memory in posttraumatic stress disorder. Behav Res Ther 2008; 46: 154-61.

35 van Harmelen AL, de Jong PJ, Glashouwer KA, Spinhoven P, Penninx BWJH, Elzinga BM. Child abuse and negative explicit and automatic selfassociations: the cognitive scars of emotional maltreatment. Behav Res Ther 2010; 48: 486-94.

$36 \mathrm{Kim} \mathrm{J,} \mathrm{Cicchetti} \mathrm{D.} \mathrm{Social} \mathrm{self-efficacy} \mathrm{and} \mathrm{behavior} \mathrm{problems} \mathrm{in} \mathrm{maltreated}$ and nonmaltreated children. J Clin Child Adolesc Psychol 2003; 32: 106-17.

37 Wright MOD, Crawford E, Del Castillo D. Childhood emotional maltreatment and later psychological distress among college students: the mediating role of maladaptive schemas. Child Abus Negl 2009; 33: 59-68.

38 Raes $F$, Hermans $D$. On the mediating role of subtypes of rumination in the relationship between childhood emotional abuse and depressed mood: brooding versus reflection. Depress Anxiety. 2008; 25: 1067-70. 